\title{
Voltammetry as a Tool to Monitor the Aggregation Behavior of a Zwitterionic Ferrocene Surfactant
}

\author{
Stefan Bitter, ${ }^{\mathrm{a}}$ Markus Schilling, ${ }^{\mathrm{a}}$ Jan P. Brahm, ${ }^{\mathrm{a}}$ Sebastian Polarz, ${ }^{\mathrm{a}, \mathrm{b} *}$ and Rainer F. Winter ${ }^{\mathrm{a} *}$ \\ aDepartment of Chemistry, University of Konstanz, Universitätsstrasse 10, 78457 Konstanz (Germany) \\ ${ }^{b}$ Institute of Inorganic Chemistry, Leibniz-University Hannover, Callinstrasse 9, 30167 Hannover (Germany) \\ Keywords: Ferro-Self-Assembly, Ferrocene, Cyclic Voltammetry, Amphiphiles, Redox-Stimulus \\ E-mail: sebastian.polarz@aca.uni-hannover.de \\ rainer.winter@uni-konstanz.de
}




\section{Supporting Information}

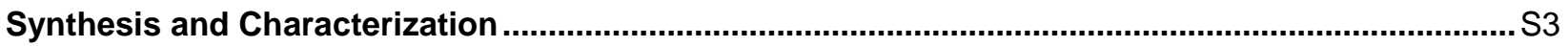

Hexyltriphenylphosphonium bromide $\left(\mathrm{Ph}_{3} \mathrm{PHexyl}^{+} \mathrm{Br}\right)$. ..................................................... 3

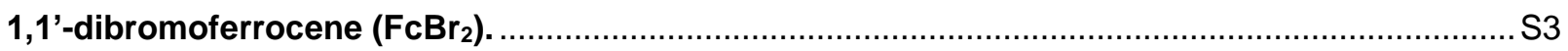

1-formyl-1'-bromoferrocene (FcBrCHO).

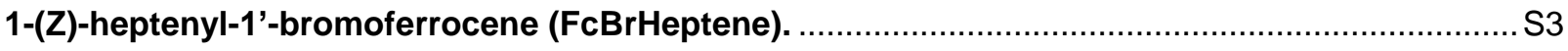

1-(Z)-heptenyl-1'-dimethylaminomethylferrocene (FcNMe ${ }_{2}$ Heptene). ......................................... 44

1-(Z)-heptenyl-1'-dimethylammoniummethyl-(3-sulfopropyl)-ferrocene (FcNMe $\mathrm{SO}_{3} \mathrm{Heptene,} \mathrm{1).S4}$

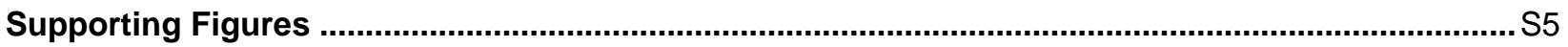

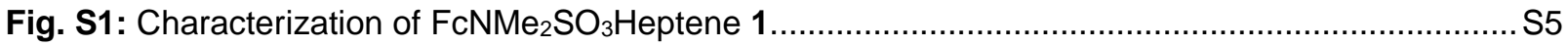

Fig. S2: Scan rate dependence of the redox-processes of 1 at $c=5.2 \mathrm{mM} 240 \mathrm{~min}$ after sample preparation.

Fig. S3: CV overlay of 1 at the lowest investigated concentration of $0.24 \mathrm{mM}$ with the capacitive background current.

Fig. S4: Square Wave voltammogram of 1 recorded in $0.01 \mathrm{M} \mathrm{KPF}_{6}$ at a concentration $c=0.24 \mathrm{mM}$. . S7

Fig. S5: Plots of $i_{\mathrm{p}, \mathrm{a}(1)}$ and $i_{\mathrm{p}, \mathrm{a}(2)}$ of $\mathbf{1}$ as a function of concentration S8

Fig. S6: Square Wave voltammograms of 1 recorded in $0.01 \mathrm{M}^{2}$ aqueous $\mathrm{KPF}_{6}$ and concentrations of $c$ $=1.84 \mathrm{mM}$ (top panel), $c=3.71 \mathrm{mM}$ (middle panel) and $c=7.41 \mathrm{mM}$ (bottom panel). S9

Fig. S7: $S W V s$ of 1 in $0.01 \mathrm{M}_{\text {aqueous }} \mathrm{KPF}_{6}$ recorded at different frequencies. S11 


\section{Synthesis and Characterization}

\section{Hexyltriphenylphosphonium bromide $\left(\mathrm{Ph}_{3} \mathrm{PHexyl}^{+} \mathrm{Br}^{-}\right)$.}

1-Bromo-hexane (6.0 mL, $42.4 \mathrm{mmol}, 1$ equiv) and $\mathrm{PPh}_{3}(11.55 \mathrm{~g}, 44.5 \mathrm{mmol}, 1.05$ equiv) were dissolved in $70 \mathrm{~mL}$ of toluene. The solution was heated to reflux for $15 \mathrm{~h}$. After cooling to room temperature, a white precipitate was obtained. It was filtered, washed three times with $20 \mathrm{~mL}$ of pentane, and dried in vacuo. $\mathrm{Ph}_{3} \mathrm{PHexyl}+\mathrm{Br}^{-}(8.44 \mathrm{~g}, 19.08 \mathrm{mmol}, 45 \%)$ was obtained as a colourless solid. ${ }^{1} \mathrm{H} \mathrm{NMR}\left(400 \mathrm{MHz}, \mathrm{CDCl}_{3}\right)$ : ס 7.91-7.83 (m, 6H, Ph-H), 7.81-7.74 (m, 3H, Ph-H), 7.73-7.66 (m, 6H, Ph-H), 3.91-3.82 (m, 2H, $\left.\mathrm{PCH}_{2}\right)$, 1.60-1.67 (m, $\left.4 \mathrm{H}, \mathrm{CH}_{2}\right), 1.26-1.18\left(\mathrm{~m}, 4 \mathrm{H}, \mathrm{CH}_{2}\right), 0.82\left(\mathrm{t},{ }^{3} \mathrm{JH}=6.9 \mathrm{~Hz}, 3 \mathrm{H}, \mathrm{CH}_{3}\right)$.

\section{1,1'-dibromoferrocene $\left(\mathrm{FcBr}_{2}\right)$.}

A solution of ferrocene (10 g, $53.75 \mathrm{mmol}, 1$ eq.), $n$-hexane ( $400 \mathrm{~mL}$ ) and TMEDA (19 mL, $125.24 \mathrm{mmol}$, 2.33 eq.) was stirred in a dried $1 \mathrm{~L}$ schlenk flask and cooled to $0{ }^{\circ} \mathrm{C}$. Then $1.6 \mathrm{M} \mathrm{n}$-BuLi in hexane $(72 \mathrm{~mL}$, $125.24 \mathrm{mmol}, 2.33$ eq.) was added dropwise and the suspension was raised to room temperature over night. The orange precipitate was filtered, re-suspended in diethyl ether $(350 \mathrm{~mL})$ and cooled to $-78{ }^{\circ} \mathrm{C}$ and a solution of $13.5 \mathrm{~mL}$ tetrabromoethane (TBE) $(115.56 \mathrm{mmol}, 2.15$ eq.) in $80 \mathrm{~mL}$ diethyl ether was added dropwise. The solution was raised to ambient temperature over night. The dark red solution was decanted and quenched with $100 \mathrm{~mL}$ of water. After solvent removal the dark orange solid was dissolved in $300 \mathrm{~mL}$ hexane and filtered trough celite and then washed subsequently with sat. aq. $\mathrm{FeCl}_{3}(\mathrm{ca} .3 \times 100 \mathrm{~mL}$ ). The organic phase was extracted with water, dried over $\mathrm{MgSO}_{4}$ and the solvent was removed in vacuo. Pure orange crystalline $\mathrm{FcBr}_{2}$ was obtained after recrystallization from $\mathrm{MeOH}$ in $59 \%$ yield $(10.89 \mathrm{~g}, 31.67 \mathrm{mmol})$. ${ }^{1} \mathrm{H}$ NMR $\left(400 \mathrm{MHz}, \mathrm{CDCl}_{3}\right): \delta 4.42\left(\mathrm{vt},{ }^{3} \mathrm{JHH}_{\mathrm{HH}}=1.9 \mathrm{~Hz}, 4 \mathrm{H}, \mathrm{Cp}-\mathrm{H}\right), 4.17$ (vt, $\left.{ }^{3} \mathrm{JHH}_{\mathrm{HH}}=1.9 \mathrm{~Hz}, 4 \mathrm{H}, \mathrm{Cp}-\mathrm{H}\right)$.

\section{1-formyl-1'-bromoferrocene ( $\mathrm{FcBrCHO}$ ).}

$\mathrm{FcBr}_{2}\left(9.32 \mathrm{~g}, 27.11 \mathrm{mmol}, 1\right.$ equiv) was dissolved in $120 \mathrm{~mL}$ THF and the solution was cooled to $-78^{\circ} \mathrm{C}$. Then, $16.9 \mathrm{~mL}$ of a $1.6 \mathrm{M}$ solution of $n$-BuLi in hexane $(27.11 \mathrm{mmol}, 1$ equiv) were added dropwise over a period of $20 \mathrm{~min}$. The red solution was stirred at $-78^{\circ} \mathrm{C}$ for further $30 \mathrm{~min}$. DMF $(3.3 \mathrm{~mL}, 43.4 \mathrm{mmol}$, 1.6 equiv) was added dropwise over a period of $10 \mathrm{~min}$. The mixture was stirred for further $30 \mathrm{~min}$ at $-78^{\circ} \mathrm{C}$ and for $2 \mathrm{~h}$ at room temperature. The reaction was quenched with $20 \mathrm{~mL}$ of $1 \mathrm{M} \mathrm{HCl}$ and $20 \mathrm{~mL}$ of saturated $\mathrm{NaCl}$ solution. The phases were separated, and the aqueous phase was extracted twice with $15 \mathrm{~mL}$ of diethyl ether. The combined organic phases were dried over $\mathrm{MgSO}_{4}$ and the solvent was removed in vacuo. The crude product was purified by column chromatography (1-9\% EE/PE) yielding $\mathrm{FcBrCHO}(7.40 \mathrm{~g}$, $25.26 \mathrm{mmol}, 93 \%)$ as dark red needles. ${ }^{1} \mathrm{H}$ NMR $\left(400 \mathrm{MHz}, \mathrm{CDCl}_{3}\right) \delta 9.99(\mathrm{~s}, 1 \mathrm{H}, \mathrm{CHO}), 4.84$ (vt, ${ }^{3} \mathrm{JHH}_{\mathrm{HH}} 1.9$ $\mathrm{Hz}, 2 \mathrm{H}, \mathrm{Cp}-\mathrm{H}), 4.63\left(\mathrm{vt},{ }^{3} \mathrm{JHH}_{\mathrm{HH}}=1.9 \mathrm{~Hz}, 2 \mathrm{H}, \mathrm{Cp}-\mathrm{H}\right), 4.52\left(\mathrm{vt},{ }^{3} \mathrm{~J}_{\mathrm{HH}}=1.9 \mathrm{~Hz}, 2 \mathrm{H}, \mathrm{Cp}-\mathrm{H}\right), 4.21\left(\mathrm{vt},{ }^{3} J_{\mathrm{HH}}=1.9 \mathrm{~Hz}\right.$, $2 \mathrm{H}, \mathrm{Cp}-\mathrm{H})$.

\section{1-(Z)-heptenyl-1'-bromoferrocene (FcBrHeptene).}

$\mathrm{BrPPh}_{3} \mathrm{Hex}$ (3.25 g, $7.61 \mathrm{mmol}, 1$ equiv) was dissolved in $60 \mathrm{~mL}$ of THF. After the addition of KO'Bu ( $0.85 \mathrm{~g}$, $7.61 \mathrm{mmol}, 1$ equiv) to the turbid solution, a colour change to red was observed. The solution was stirred for $1 \mathrm{~h}$. A solution of $\mathrm{FcBrCHO}(2.23 \mathrm{~g}, 7.61 \mathrm{mmol}, 1$ equiv) in $20 \mathrm{~mL}$ of THF was added dropwise over a period of $20 \mathrm{~min}$. The solution was stirred overnight. $40 \mathrm{~mL}$ of $n$-pentane were added, and the precipitate was filtered off. The solvent was removed in vacuo and the crude product was purified by column chromatography (50\% EE/PE) yielding FcBrHeptene $(2.52 \mathrm{~g}, 7.00 \mathrm{mmol}, 92 \%)$ as a brown oil. FcBrHeptene was obtained selectively in (Z)-configuration. ${ }^{1} \mathrm{H} \mathrm{NMR}\left(400 \mathrm{MHz}, \mathrm{CDCl}_{3}\right) \delta 6.06\left(\mathrm{dt},{ }^{3} \mathrm{JHH}\right.$,cis $=11.3 \mathrm{~Hz}$, $\left.{ }^{4} J_{\mathrm{HH}}=1.8 \mathrm{~Hz}, 1 \mathrm{H}, \mathrm{CpC}-\mathrm{H}\right), 5.55\left(\mathrm{dt},{ }^{3} \mathrm{JHH}_{\mathrm{HH}}\right.$,is $\left.=11.3 \mathrm{~Hz},{ }^{3} \mathrm{~J}_{\mathrm{HH}}=7.2 \mathrm{~Hz}, 1 \mathrm{H}, \mathrm{C}=\mathrm{CH}\right), 4.32\left(\mathrm{vt},{ }^{3} J_{\mathrm{HH}}=1.9 \mathrm{~Hz}\right.$, $2 \mathrm{H}, \mathrm{Cp}-\mathrm{H}), 4.30\left(\mathrm{vt},{ }^{3} \mathrm{JHH}_{\mathrm{HH}}=1.9 \mathrm{~Hz}, 2 \mathrm{H}, \mathrm{Cp}-\mathrm{H}\right), 4.26\left(\mathrm{vt},{ }^{3} \mathrm{JHH}_{\mathrm{HH}}=1.9 \mathrm{~Hz}, 2 \mathrm{H}, \mathrm{Cp}-\mathrm{H}\right), 4.05\left(\mathrm{vt},{ }^{3} \mathrm{JHH}_{\mathrm{HH}}=1.9 \mathrm{~Hz}, 2 \mathrm{H}\right.$, $\mathrm{Cp}-\mathrm{H}), 2.25-2.22\left(\mathrm{~m}, 2 \mathrm{H}, \mathrm{CH}_{2}\right), 1.51-1.42\left(\mathrm{~m}, 2 \mathrm{H}, \mathrm{CH}_{2}\right), 1.39-1.32\left(\mathrm{~m}, 4 \mathrm{H}, \mathrm{CH}_{2}\right), 0.92\left(\mathrm{t},{ }^{3} \mathrm{JHH}_{\mathrm{H}}=7.2 \mathrm{~Hz}, \mathrm{CH}_{3}\right)$. 


\section{1-(Z)-heptenyl-1'-dimethylaminomethylferrocene (FcNMe 2 Heptene).}

FcBrHeptene (1.14 g, $3.16 \mathrm{mmol}, 1$ equiv) was dissolved in $50 \mathrm{~mL}$ of THF and the solution was cooled to $78^{\circ} \mathrm{C}$. Then, $2.0 \mathrm{~mL}$ of a $1.6 \mathrm{M}$ solution of $n$-BuLi in hexane $(3.16 \mathrm{mmol}, 1$ equiv) were added dropwise over a period of $5 \mathrm{~min}$. The solution was stirred at $-78^{\circ} \mathrm{C}$ for another $10 \mathrm{~min}$. Eschenmoser's salt $(585 \mathrm{mg}$, $3.16 \mathrm{mmol}, 1$ equiv) was added and the temperature was kept at $-78^{\circ} \mathrm{C}$ for one hour. Then, $20 \mathrm{~mL}$ of distilled water and $40 \mathrm{~mL}$ of ethyl acetate were added. The phases were separated, and the aqueous phase was extracted twice with ethyl acetate. The combined organic layers were washed with brine and dried over $\mathrm{MgSO}_{4}$. The solvent was removed in vacuo and the crude product was purified by column chromatography (5\% NEt $3 / \mathrm{PE})$ yielding $400 \mathrm{mg}$ of $\mathrm{FcNMe}_{2} \mathrm{Heptene}(1.18 \mathrm{mmol}, 37 \%)$ as a brown oil. ${ }^{1} \mathrm{H} \mathrm{NMR}(400 \mathrm{MHz}$, $\left.\mathrm{CDCl}_{3}\right) \delta 6.02\left(\mathrm{~d},{ }^{3} J_{\mathrm{HH}, \mathrm{cis}}=11.5 \mathrm{~Hz}, 1 \mathrm{H}, \mathrm{CpC}-\mathrm{H}\right), 5.51$ (dt, ${ }^{3} J_{\mathrm{HH}, \mathrm{cis}}=11.5 \mathrm{~Hz},{ }^{3} \mathrm{JHH}_{\mathrm{HH}}=7.2 \mathrm{~Hz}, 1 \mathrm{H}, \mathrm{C}=\mathrm{CH}$ ), 4.28-4.24 (m, 2H, Cp-H), 4.18-4.15 (m, 2H, Cp-H), 4.11-4.06 (m, 4H, Cp-H), $3.25\left(\mathrm{~s}, 2 \mathrm{H}, \mathrm{NCH}_{2}\right), 2.26-2.24$ $\left(\mathrm{m}, 2 \mathrm{H}, \mathrm{CH}_{2}\right), 2.18\left(\mathrm{~s}, 6 \mathrm{H}, \mathrm{NCH}_{3}\right), 1.52-1.42\left(\mathrm{~m}, 2 \mathrm{H}, \mathrm{CH}_{2}\right), 1.41-1.34\left(\mathrm{~m}, 2 \mathrm{H}, \mathrm{CH}_{2}\right), 0.94\left(\mathrm{t},{ }^{3} \mathrm{~J}_{\mathrm{HH}}=7.0 \mathrm{~Hz}\right.$, $\left.\mathrm{CH}_{3}\right)$.

\section{1-(Z)-heptenyl-1'-dimethylammoniummethyl-(3-sulfopropyl)-ferrocene ( $\left.\mathrm{FCNMe}_{2} \mathrm{SO}_{3} \mathrm{Heptene}_{1}, 1\right)$.}

A solution of $\mathrm{FcNMe}_{2} \mathrm{Heptene}(400 \mathrm{mg}, 1.18 \mathrm{mmol}, 1$ equiv) and 1,3-propane sultone (171 mg, $1.40 \mathrm{mmol}$, 1.2 equiv) in $40 \mathrm{~mL}$ of acetonitrile was heated to reflux for $15 \mathrm{~h}$. After cooling to room temperature, the

solvent was removed in vacuo. The orange precipitate was washed four times with toluene $(3 \mathrm{~mL})$ yielding $380 \mathrm{mg}$ of $\mathrm{FcNMe}_{2} \mathrm{SO}_{3} \mathrm{Heptene}(0.82 \mathrm{mmol}, 70 \%)$. ${ }^{1} \mathrm{H}$ NMR $(400 \mathrm{MHz}, \mathrm{MeOD}) \delta 6.10\left(\mathrm{dt},{ }^{3} \mathrm{JH}, \mathrm{His}=11.4 \mathrm{~Hz}\right.$, $\left.{ }^{4} J_{\mathrm{HH}}=1.7 \mathrm{~Hz}, 1 \mathrm{H}, \mathrm{H}-12\right), 5.61\left(\mathrm{dt},{ }^{3} J_{\mathrm{HH}, \mathrm{cis}}=11.4 \mathrm{~Hz},{ }^{3} J_{\mathrm{HH}}=7.3 \mathrm{~Hz}, 1 \mathrm{H}, \mathrm{H}-13\right), 4.48\left(\mathrm{vt},{ }^{3} J_{\mathrm{HH}}=1.9 \mathrm{~Hz}, 2 \mathrm{H}\right.$, $\mathrm{H}-7$ ), 4.45 (vt, $\left.{ }^{3} \mathrm{JHH}_{\mathrm{HH}} 1.9 \mathrm{~Hz}, 2 \mathrm{H}, \mathrm{H}-10\right), 4.38$ (vt, $\left.{ }^{3} \mathrm{JHH}_{\mathrm{HH}}=1.9 \mathrm{~Hz}, 2 \mathrm{H}, \mathrm{H}-8\right), 4.37$ (s, $\left.2 \mathrm{H}, \mathrm{H}-5\right), 4.34$ (vt, $\left.{ }^{3} J_{\mathrm{HH}}=1.9 \mathrm{~Hz}, 2 \mathrm{H}, \mathrm{H}-9\right), 3.45-3.39(\mathrm{~m}, 2 \mathrm{H}, \mathrm{H}-3), 2.96(\mathrm{~s}, 6 \mathrm{H}, \mathrm{H}-4), 2.88\left(\mathrm{t},{ }^{3} \mathrm{~J}_{\mathrm{HH}}=6.9 \mathrm{~Hz}, 2 \mathrm{H}, \mathrm{H}-1\right), 2.31-2.15$ (m, 4H, H-2, H-14), 1.54-1.45 (m, 2H, H-15), 1.43-1.35 (m, 4H, H-16, H-17), $0.95\left(\mathrm{t},{ }^{3} \mathrm{JHH}_{\mathrm{H}}=7.1 \mathrm{~Hz}, 3 \mathrm{H}, \mathrm{H}-\right.$ 18). ${ }^{13} \mathrm{C}$ NMR (151 MHz, CDCl 3 ) $\delta 131.67$ (s, C-13), 124.53 (s, C-12), 84.07 (s, C-11), 72.86 (s, C-7), 72.35 (s, C-9), 71.97 (s, C-6), 70.36 (s, C-10), 70.15 (s, C-8), 65.68 (s, C-5), 63.21 (s, C-3), 49.55 (s, C-4), 48.07 (s, C-1), 31.80 (s, C-16), 29.53 (s, C-15), 29.19 (s, C-14), 22.74 (s, C-17), 19.73 (s, C-2), 14.25 (s, C-18). ESIMS: [gmol-1]: $(4 \mathrm{M}+\mathrm{Na}+\mathrm{H})^{+}=\left(\mathrm{C}_{92} \mathrm{H}_{141} \mathrm{Fe}_{4} \mathrm{~N}_{4} \mathrm{O}_{12} \mathrm{~S}_{4} \mathrm{Na}\right)^{+}$calc.: 1868.67, found: $1868.67 ;(4 \mathrm{M}+\mathrm{Na})^{+}=$ $\left(\mathrm{C}_{92} \mathrm{H}_{140} \mathrm{Fe}_{4} \mathrm{~N}_{4} \mathrm{O}_{12} \mathrm{~S}_{4} \mathrm{Na}\right)^{+}$calc.: 1867.66 , found: $1867.66 ;(3 \mathrm{M}+\mathrm{Na})^{+}=\left(\mathrm{C}_{69} \mathrm{H}_{105} \mathrm{Fe}_{3} \mathrm{~N}_{3} \mathrm{O}_{9} \mathrm{~S}_{3} \mathrm{Na}\right)^{+}$calc.: 1406.50, found: 1406.50; $(2 \mathrm{M}+\mathrm{Na})^{+}=\left(\mathrm{C}_{46} \mathrm{H}_{70} \mathrm{Fe}_{2} \mathrm{~N}_{2} \mathrm{O}_{6} \mathrm{~S}_{2} \mathrm{Na}\right)^{+}$calc.: 945.33, found: 945.33; $(2 \mathrm{M}+\mathrm{H})^{+}=$ $\left(\mathrm{C}_{46} \mathrm{H}_{71} \mathrm{Fe}_{2} \mathrm{~N}_{2} \mathrm{O}_{6} \mathrm{~S}_{2}\right)^{+}$calc.: 923.34 , found: $923.34 ;(\mathrm{M}+\mathrm{K})^{+}=\left(\mathrm{C}_{23} \mathrm{H}_{35} \mathrm{FeNO}_{3} \mathrm{SK}\right)^{+}$calc.: 500.13 , found: 500.13 ; $(\mathrm{M}+\mathrm{Na})^{+}=\left(\mathrm{C}_{23} \mathrm{H}_{35} \mathrm{FeNO}{ }_{3} \mathrm{SNa}\right)^{+}$calc.: 484.16 , found: $484.15 ;(\mathrm{M}+\mathrm{H})^{+}=\left(\mathrm{C}_{23} \mathrm{H}_{36} \mathrm{FeNO}_{3} \mathrm{~S}\right)^{+}$calc.: 462.18 , found: $462.17 ;(\mathrm{M})^{+}=\left(\mathrm{C}_{23} \mathrm{H}_{35} \mathrm{FeNO}_{3} \mathrm{~S}\right)^{+}$calc.: 461.17, found: $461.17 ;\left(\mathrm{M}-\mathrm{NMe}_{2} \mathrm{SO}_{3}\right)^{+}=\left(\mathrm{C}_{18} \mathrm{H}_{23} \mathrm{Fe}\right)^{+}$calc.: 295.11, found: 295.11. IR (powder): 3067, 3037, 2955, 2918, 2853, 1638. UV-Vis (MeCN): = $225 \mathrm{M}^{-1} \mathrm{~cm}^{-1}$. 


\section{Supporting Figures}

Fig. S1: Characterization of FcNMe $2 \mathrm{SO}_{3}$ Heptene 1.

(a) ${ }^{1} \mathrm{H}$ NMR of 1.
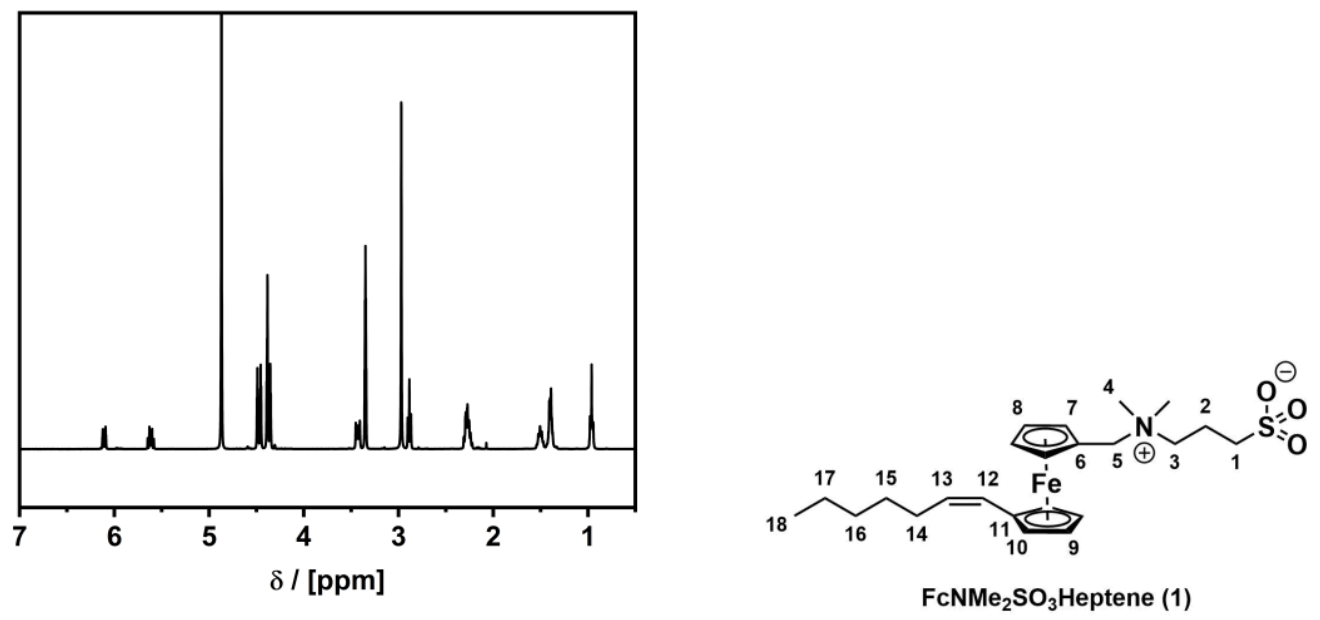

${ }^{1} \mathbf{H}$ NMR $(400 \mathrm{MHz}, \mathrm{MeOD}) \delta[\mathrm{ppm}]: 6.10\left(\mathrm{dt},{ }^{3} \mathrm{JHH}_{\mathrm{H}, \mathrm{cis}}=11.4 \mathrm{~Hz},{ }^{4} \mathrm{~J}_{\mathrm{HH}}=1.7 \mathrm{~Hz}, 1 \mathrm{H}, \mathbf{H}-12\right), 5.61$ (dt, $\left.{ }^{3} \mathrm{JHH}_{\mathrm{H}, \mathrm{cis}}=11.4 \mathrm{~Hz},{ }^{3} \mathrm{JHH}_{\mathrm{HH}}=7.3 \mathrm{~Hz}, 1 \mathrm{H}, \mathbf{H - 1 3}\right), 4.48\left(\mathrm{vt},{ }^{3} \mathrm{JHH}_{\mathrm{HH}}=1.9 \mathrm{~Hz}, 2 \mathrm{H}, \mathbf{H}-7\right), 4.45\left(\mathrm{vt},{ }^{3} \mathrm{JHH}_{\mathrm{HH}}=1.9 \mathrm{~Hz}, 2 \mathrm{H}, \mathbf{H}-\right.$ 10), 4.38 (vt, $\left.{ }^{3} \mathrm{JHH}_{\mathrm{HH}}=1.9 \mathrm{~Hz}, 2 \mathrm{H}, \mathbf{H}-8\right), 4.37(\mathrm{~s}, 2 \mathrm{H}, \mathbf{H}-5), 4.34\left(\mathrm{vt},{ }^{3} \mathrm{JHH}_{\mathrm{HH}}=1.9 \mathrm{~Hz}, 2 \mathrm{H}, \mathbf{H}-9\right), 3.45-3.39(\mathrm{~m}, 2 \mathrm{H}$, H-3), $2.96(\mathrm{~s}, 6 \mathrm{H}, \mathbf{H}-4), 2.88\left(\mathrm{t},{ }^{3} \mathrm{JHH}_{\mathrm{HH}}=6.9 \mathrm{~Hz}, 2 \mathrm{H}, \mathbf{H}-1\right), 2.31-2.15(\mathrm{~m}, 4 \mathrm{H}, \mathbf{H}-2, \mathbf{H}-14), 1.54-1.45(\mathrm{~m}, 2 \mathrm{H}$, H-15), 1.43-1.35 (m, 4H, H-16, H-17), $0.95\left(t,{ }^{3} \mathrm{HH}=7.1 \mathrm{~Hz}, 3 \mathrm{H}, \mathbf{H}-18\right)$.

(b) ${ }^{13} \mathrm{C}\left\{{ }^{1} \mathrm{H}\right\}$ NMR of 1.
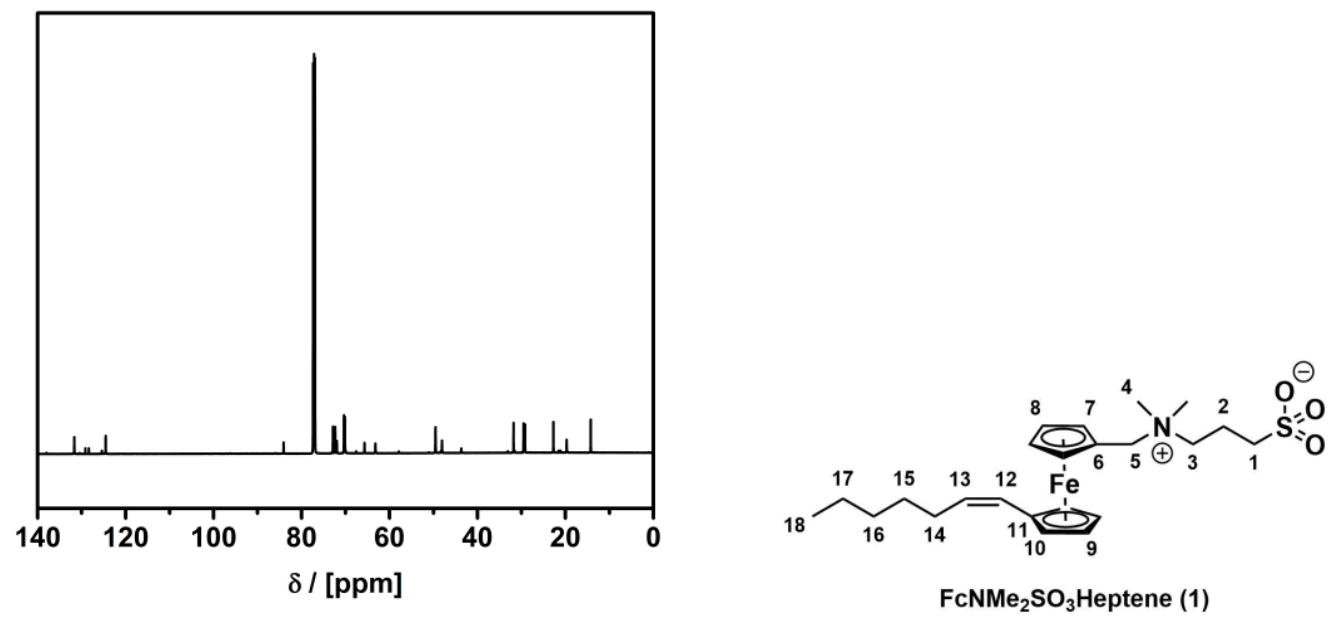

${ }^{13} \mathrm{C}$ NMR $\left(151 \mathrm{MHz}, \mathrm{CDCl}_{3}\right.$ ) $\delta$ [ppm]: 131.67 (s, C-13), 124.53 (s, C-12), 84.07 (s, C-11), 72.86 (s, C-7), 72.35 (s, C-9), 71.97 (s, C-6), 70.36 (s, C-10), 70.15 (s, C-8), 65.68 (s, C-5), 63.21 (s, C-3), 49.55 (s, C-4), 48.07 (s, C-1), 31.80 (s, C-16), 29.53 (s, C-15), 29.19 (s, C-14), 22.74 (s, C-17), 19.73 (s, C-2), 14.25 (s, C18). 
Fig. S2: Scan rate dependence of the redox-processes of 1 at $c=5.2 \mathrm{mM} 240 \mathrm{~min}$ after sample preparation.

a)

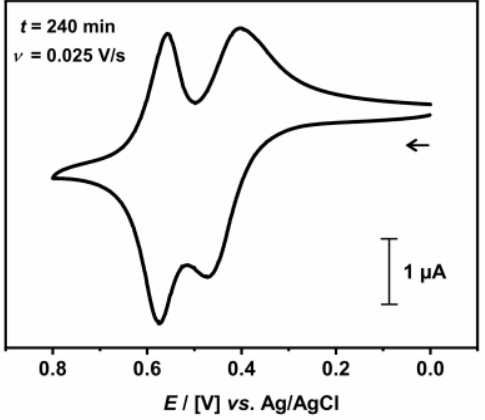

b)

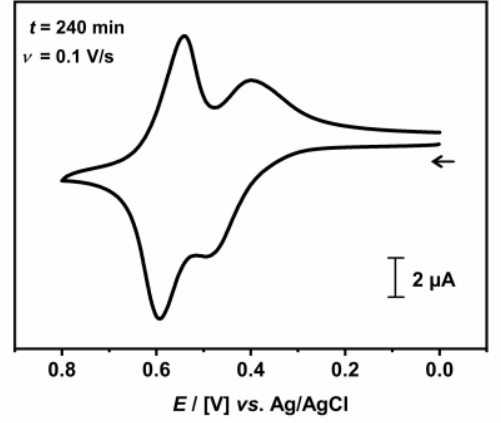

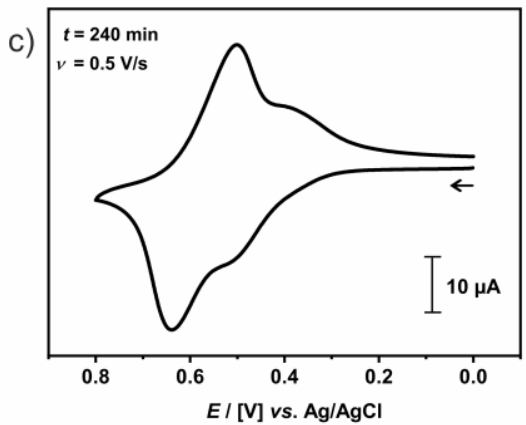

$\mathrm{CV}$ s of 1 recorded in $0.01 \mathrm{M}$ aqueous $\mathrm{KPF}_{6}, 240 \mathrm{~min}$ after sample preparation. Different scan rates, at a) $v$ $=0.025 \mathrm{~V} / \mathrm{s}, \mathrm{b}) v=0.1 \mathrm{~V} / \mathrm{s}$ and c) $v=0.5 \mathrm{~V} / \mathrm{s}$.

Fig. S3: CV overlay of 1 at the lowest investigated concentration of $0.24 \mathrm{mM}$ with the capacitive background current.

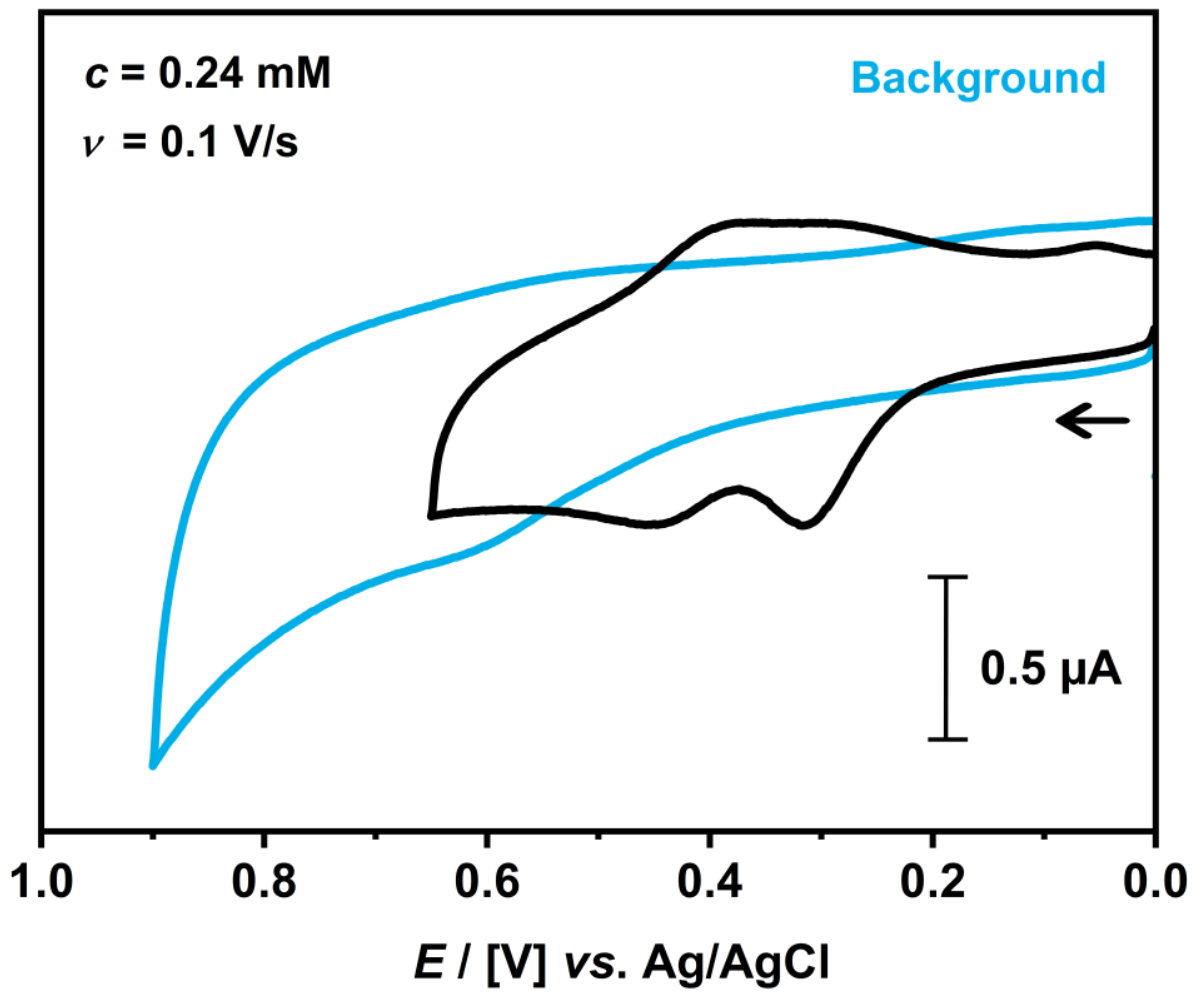

$\mathrm{CV}$ of 1 at the lowest investigated concentration of $0.24 \mathrm{mM}$ (black line) compared to the capacitive background current (blue line) measured in $0.01 \mathrm{M} \mathrm{KPF}_{6}$ at a scan rate $v$ of $0.1 \mathrm{~V} / \mathrm{s}$. 
Fig. S4: Square Wave voltammogram of 1 recorded in $0.01 \mathrm{M} \mathrm{KPF}_{6}$ at a concentration $c=0.24 \mathrm{mM}$.

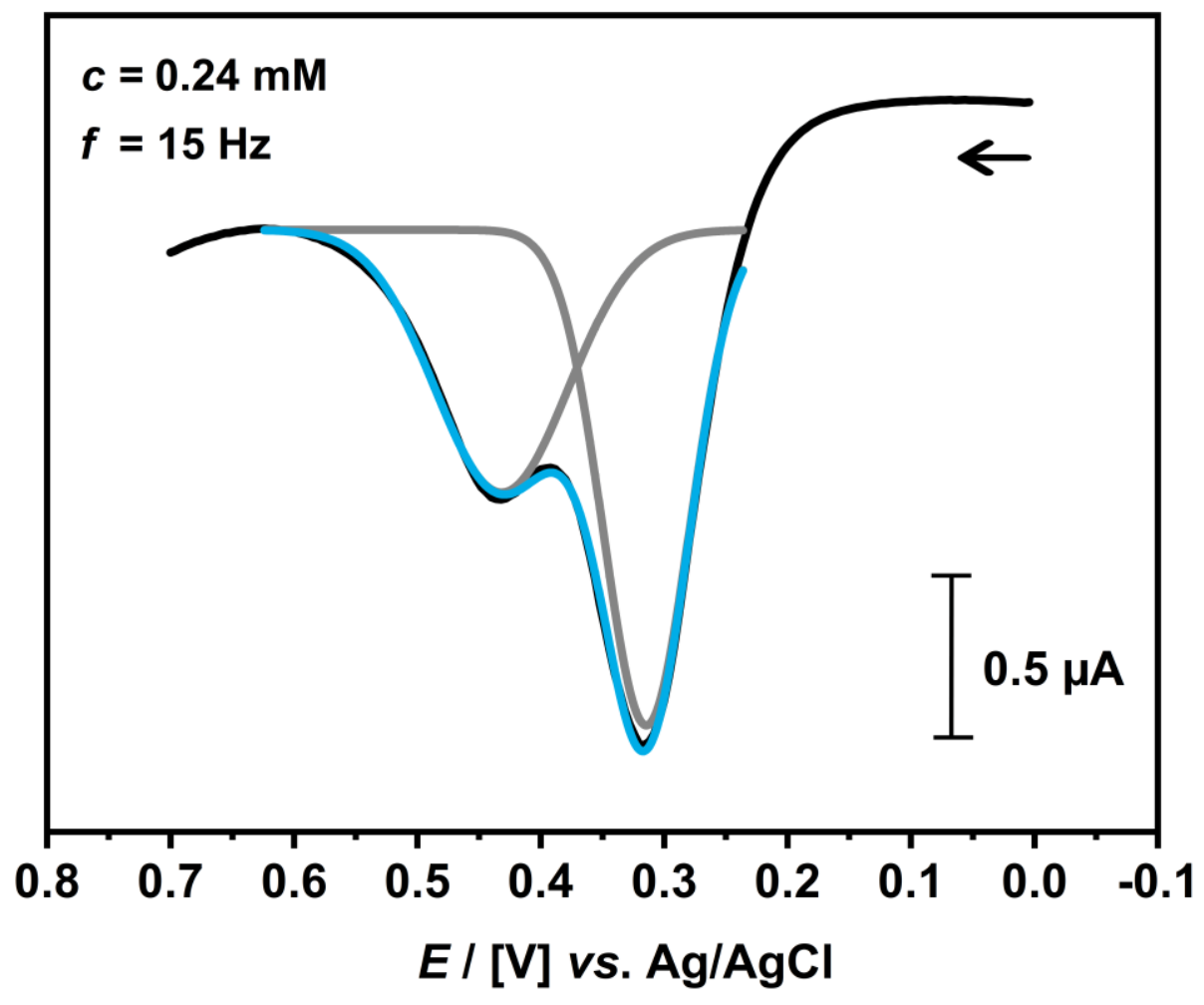


Fig. S5: Plots of $i_{p, a(1)}$ and $i_{p, a(2)}$ of 1 as a function of concentration.

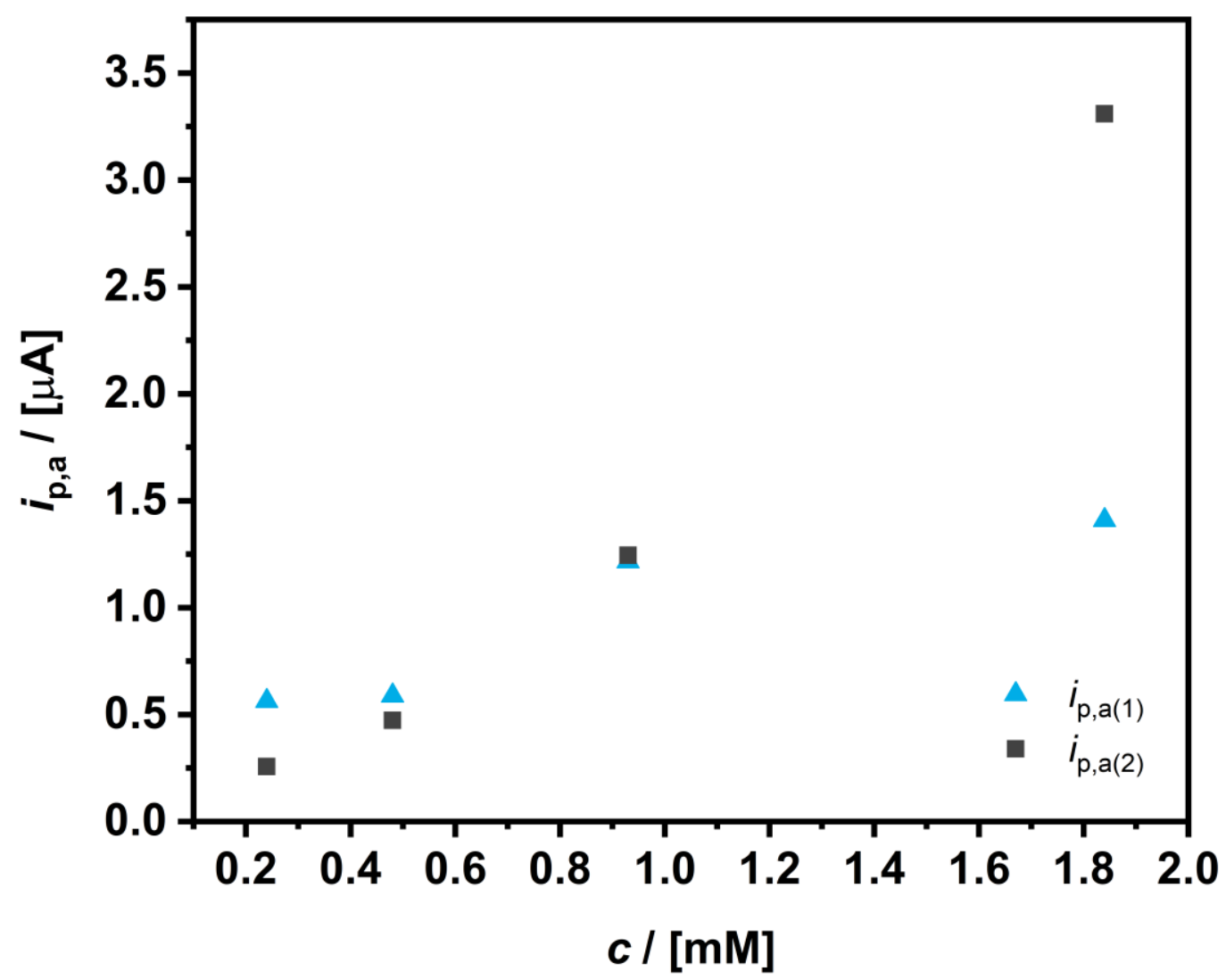


Fig. S6: Square Wave voltammograms of 1 recorded in $0.01 \mathrm{M}$ aqueous $\mathrm{KPF}_{6}$ and concentrations of $c=1.84 \mathrm{mM}$ (top panel), $c=3.71 \mathrm{mM}$ (middle panel) and $c=7.41 \mathrm{mM}$ (bottom panel).
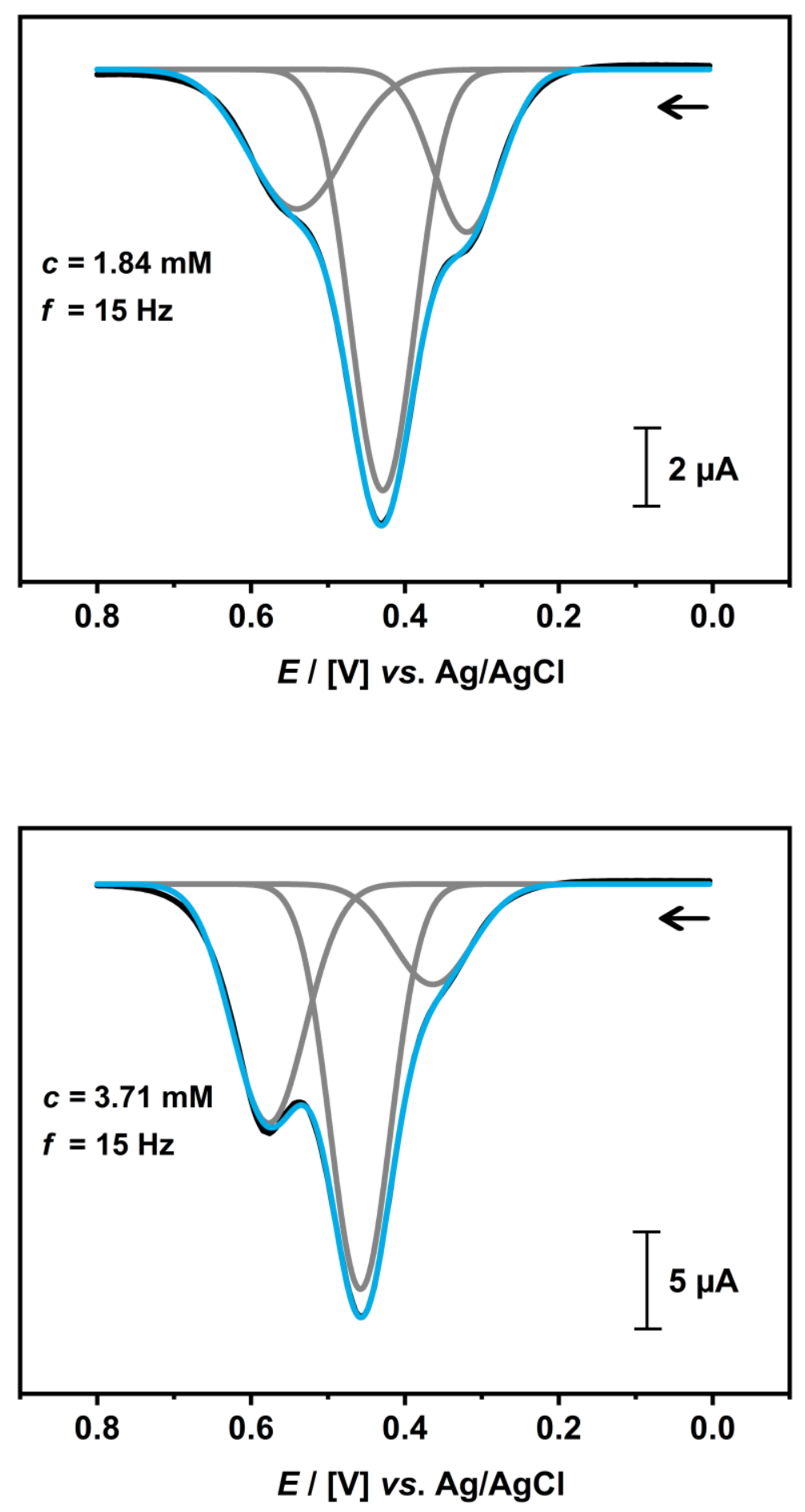


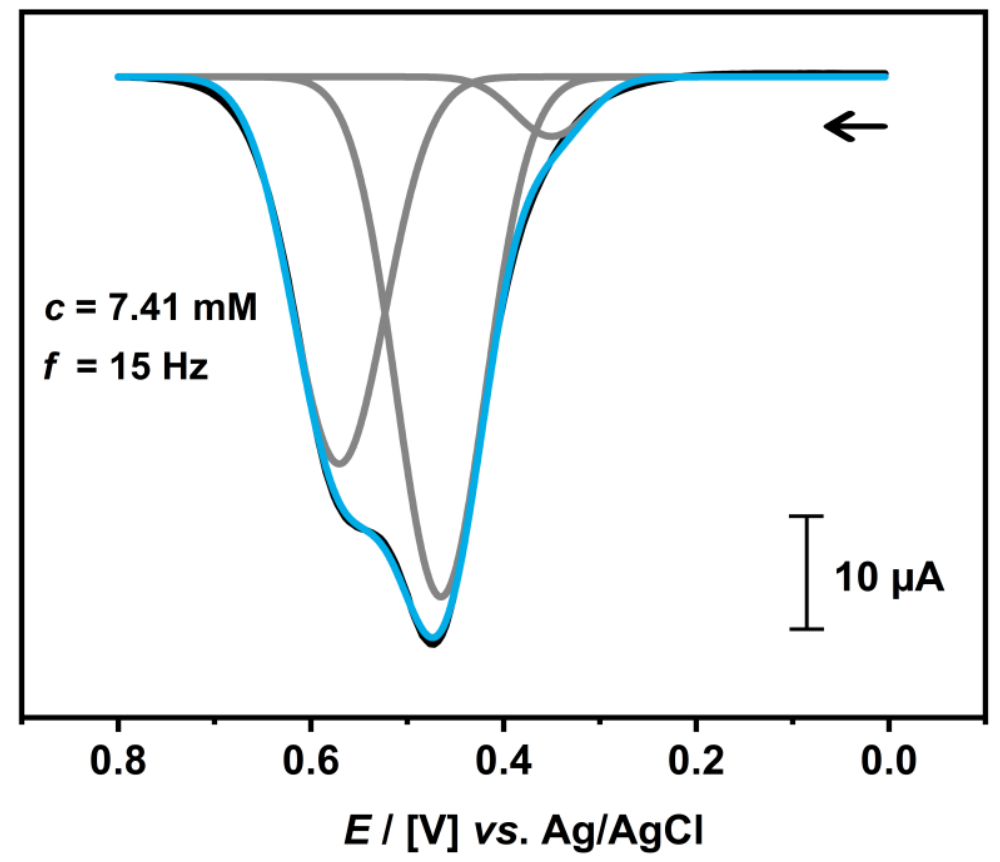


Fig. S7: SWVs of 1 in $0.01 \mathrm{M}$ aqueous $\mathrm{KPF}_{6}$ recorded at different frequencies.

$30 \mathrm{~Hz}$

$15 \mathrm{~Hz}$

$5 \mathrm{~Hz}$

Freely Diffusing Monomer (2)

Aggregate (3') " Adsorbed Monomer (1)
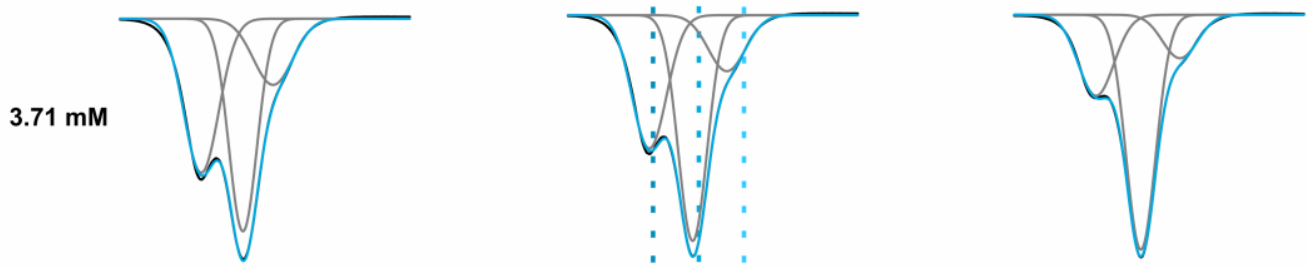

$\begin{array}{lllllllllllllll}1.0 & 0.9 & 0.8 & 0.7 & 0.6 & 0.5 & 0.4 & 0.3 & 0.2 & 0.1 & 0.0 & 0.1\end{array}$

$\begin{array}{lllllllllllllll}1.0 & 0.9 & 0.8 & 0.7 & 0.6 & 0.5 & 0.4 & 0.3 & 0.2 & 0.1 & 0.0 & 0.1\end{array}$
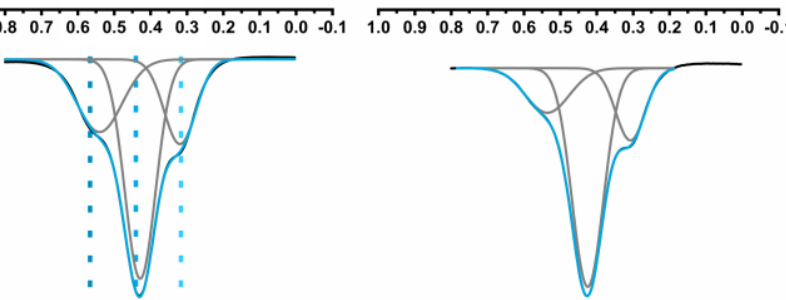

$\begin{array}{lllllllllllllllll}1.0 & 0.9 & 0.8 & 0.7 & 0.6 & 0.5 & 0.4 & 0.3 & 0.2 & 0.1 & 0.0 & 0.1\end{array}$

$\begin{array}{lllllllllllllllllllllllllllllllll}1.0 & 0.9 & 0.8 & 0.7 & 0.6 & 0.5 & 0.4 & 0.3 & 0.2 & 0.1 & 0.0 & -0.1 & 1.0 & 0.9 & 0.8 & 0.7 & 0.6 & 0.5 & 0.4 & 0.3 & 0.2 & 0.1 & 0.0 & 0.0\end{array}$

cac

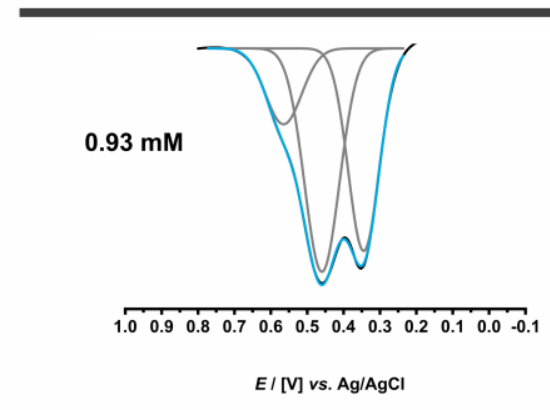

$0.48 \mathrm{mM}$
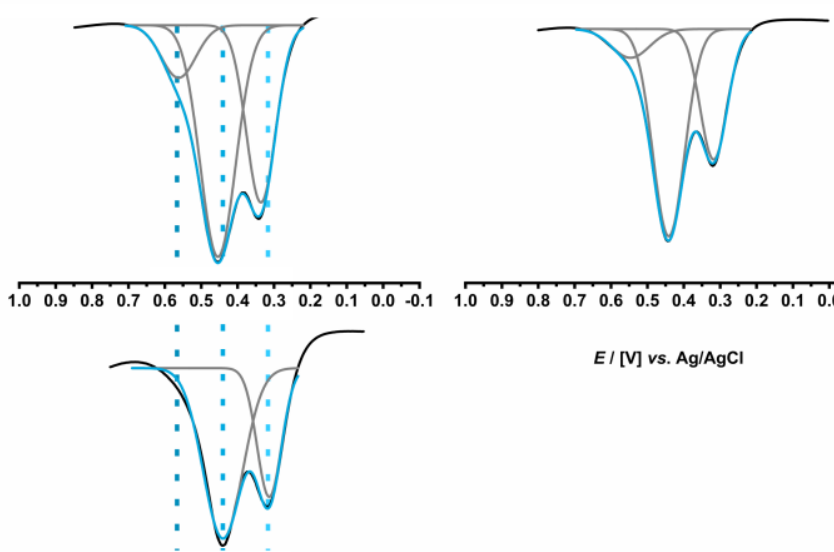

$\begin{array}{lllllllllllllllll}1.0 & 0.9 & 0.8 & 0.7 & 0.6 & 0.5 & 0.4 & 0.3 & 0.2 & 0.1 & 0.0 & -0.1\end{array}$

$E /[\mathrm{V}]$ vs. Ag/AgCl

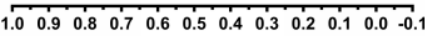

$0.24 \mathrm{mM}$

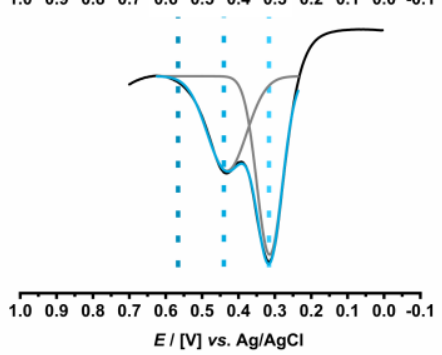

Concentration-dependent SWV measurements of 1 performed in $0.01 \mathrm{M}$ aqueous $\mathrm{KPF}_{6}$. For the sake of clarity, the respective SWVs are depicted at different current scales. Color code: Dark blue color indicates the aggregation wave, sea blue that of the freely diffusing monomers, and pale that of the adsorbed monomers. 